\title{
Perceived Importance of Breast Cancer Risk Factors: A Survey on 386 Physicians in China
}

\author{
Louis Wing-Cheong Chow,2*, Pei-Fen Fu ${ }^{3}$, Lei Guo ${ }^{4}$, Xi-Chun $\mathrm{Hu}^{5}$, Jun Jiang ${ }^{6}$, \\ Erich Ferdiansyah Lie ${ }^{1}$, Jian Liu', Xiao-Hong $\mathrm{Lu}^{2}$, Yong-Kui Lu ${ }^{8}$, Hong-Min \\ Ma $^{9}$, Qin-Guo Mo ${ }^{10}$, Yan-Xia Shi ${ }^{11}$, Kun Wang ${ }^{12,13}$, Ming-Hao Wang ${ }^{6}$, Shu Wang ${ }^{14}$, \\ Shu-Sen Wang ${ }^{15}$, Xian-Ming Wang ${ }^{16}$, Xiao-Jia Wang ${ }^{17}$, Hui-Jing Wu ${ }^{18}$, Hong-Jian \\ Yang $^{19}$, He-Rui Yao ${ }^{20}$, Yi Zhang ${ }^{6}$, Li Zhu ${ }^{21}$, on behalf of the Asian Institute of \\ Clinical Oncology (AICO) Expert Panel
}

\begin{abstract}
There are varying definitions of women at high risk of breast cancer across different institutions, and there are reports suggesting that the breast cancer risk assessment tools have not been well integrated into clinical practice. In this study, we tried to investigate the perceived importance of different breast cancer risk factors by physicians in China. A cross-sectional survey involving 386 anonymous physicians was conducted using a 20-item, 5-point Likert scale questionnaire. The Kruskal-Wallis test and post-hoc pairwise comparisons were used to compare the differences in response. Most of the respondents were either breast surgeons/specialists $(\mathrm{n}=161 ; 41.7 \%)$ or medical oncologists $(\mathrm{n}=151 ; 39.1 \%)$, and the results showed that the breast cancer risk factors were not perceived as equally important. The weighting of each risk factor also varied depending on the physician's medical specialty, location of practice, and the number of years of clinical experience. This study provides a more updated insight into the perceptions of physicians in China toward the breast cancer risk factors, as well as underlines the potential improvements in breast cancer risk assessment strategies that can be done.
\end{abstract}

Keywords: Breast cancer- risk factor- perceived importance- questionnaire- China

Asian Pac J Cancer Prev, 23 (2), 379-382

\section{Introduction}

Breast cancer screening is considered as one of the most important ways to improve the survival rates of breast cancer patients (Huang et al., 2019), especially when considering the rapid increase of breast cancer

${ }^{1}$ UNIMED Medical Institute, Hong Kong SAR, China. ${ }^{2}$ Organisation for Oncology and Translational Research, Hong Kong SAR, China. ${ }^{3}$ Department of Breast Surgery, The First Affiliated Hospital, Zhejiang University, Hangzhou, China. ${ }^{4}$ Department of Burn and Plastic Surgery, the First Affiliated Hospital of Chongqing Medical University, Chongqing, China. ${ }^{5}$ Department of Medical Oncology, Fudan University Shanghai Cancer Center, Xuhui District, Shanghai, China. ${ }^{6}$ Breast Disease Centre, Southwest Hospital, Third Military Medical University, Chongqing, China. ${ }^{7}$ Department of Breast Medical Oncology, Fujian Provincial Tumor Hospital, Fuzhou, China. ${ }^{8}$ The Department of Breast and Bone Tissue Oncology, Affiliated Tumor Hospital of Guangxi Medical University, China. ${ }^{9}$ Department of Breast Surgery, Guangzhou Women and Children's Medical Center, Guangzhou, China. ${ }^{10}$ Department of Breast Surgery, Affiliated Tumor Hospital of Guangxi Medical University, Nanning, China. ${ }^{11}$ Department of Medical Oncology, Sun Yat-sen University Cancer Center, Guangzhou, China. ${ }^{12}$ Department of Breast Cancer, Cancer Center, Guangdong Provincial People's Hospital and Guangdong Academy of Medical Sciences, Guangzhou, China. ${ }^{13}$ The Second School of Clinical Medicine, Southern Medical University, Guangzhou, China. ${ }^{14}$ Breast Center, Peking University People's Hospital, Beijing, China. ${ }^{15}$ Department of Medical Oncology, Sun Yat-Sen University Cancer Center, Guangzhou, China. ${ }^{16}$ Department of Thyroid and Breast Surgery, Shenzhen Second People's Hospital, The First Affiliated Hospital of Shenzhen University, Shenzhen, China. ${ }^{17}$ Department of Breast Medical Oncology, Cancer Hospital of the University of Chinese Academy of Sciences / Zhejiang Cancer Hospital/Institute of Cancer and Basic Medicine (ICBM), Chinese Academy of Sciences, Hangzhou, China. ${ }^{18}$ Department of Medical Oncology, Hubei Cancer Hospital, Tongji Medical College, Huazhong University of Science and Technology, Wuhan, Hubei, China. ${ }^{19}$ Department of Breast Surgery, The Cancer Hospital of the University of Chinese Academy of Sciences (Zhejiang Cancer Hospital), Institute of Basic Medicine and Cancer (IBMC), Chinese Academy of Sciences, Hangzhou, Zhejiang, China. ${ }^{20}$ Department of Oncology, Sun Yat-Sen Memorial Hospital of Sun Yat-Sen University, Guangzhou, China. ${ }^{21}$ Department of Thyroid and Breast Surgery, Shanghai General Hospital, Shanghai Jiao Tong University School of Medicine, Shanghai, China. *For Correspondence: lwcchow@ootr.org 
burden in China over the years (Feng et al., 2018). With the improved understanding of known risk factors on breast cancer and development of breast cancer risk assessment tools, there is ample opportunity for promoting breast cancer prevention and risk reduction. However, there are varying definitions of women at high risk of breast cancer across different institutions, and there are reports suggesting that the breast cancer risk assessment tools have not been well integrated into clinical practice (Livaudais-Toman et al., 2015).

In this study, we tried to investigate the perceived importance of different breast cancer risk factors by physicians in China. It was unknown whether their medical specialties, locations of practice, and the numbers of years of clinical experience would affect their perceptions toward the breast cancer risk factors, and we suspected that the differences in perceptions may affect the implementation and success of breast cancer risk assessment strategies in China. To the best of our knowledge, there are no studies that have explored this aspect before.

\section{Materials and Methods}

A cross-sectional survey involving 386 anonymous physicians from all over China was conducted in January 2021. The online survey invitation was sent to the department heads of hospitals around China and disseminated within their respective departments.

The questionnaire consisted of 20 items that are considered or hypothesized as breast cancer risk factors. A 5-point Likert scale ranging from "Least Important" to "Most Important" was used to determine the weighting of each risk factor. In addition, respondents were asked to indicate their medical specialties, cities and provinces of practice, and numbers of years of clinical experience.

Statistical analyses were performed using SPSS version 25.0. After grouping the respondents based on their medical specialties, cities and provinces of practice, and numbers of years of clinical experience, the KruskalWallis test was used to compare the differences in response between the subgroups. Post-hoc pairwise comparisons were analyzed when a statistically significant difference was found.

\section{Results}

\section{Characteristics of respondents}

Medical specialties

Most of the respondents were either breast surgeons/specialists $(\mathrm{n}=161 ; 41.7 \%)$ or medical oncologists $(n=151 ; 39.1 \%)$. There were $33(8.5 \%)$ respondents who indicated their medical specialty as general surgeon or surgical oncologist; $20(5.2 \%)$ respondents who were radiation oncologists or radiotherapists; 7 (1.8\%) respondents who were internal medicine physicians; 6 $(1.6 \%)$ respondents who did not describe their medical specialties; $3(0.8 \%)$ respondents who were cardiothoracic surgeons; 2 (0.5\%) respondents who were pathologists; and A\&E physician, Chinese medicine practitioner, and plastic surgeon with $1(0.3 \%)$ respondent each.

\section{Chinese city tier system}

When grouped based on the hierarchical classification (with Tier 1 cities being the most developed areas of the country), there were $174(45.1 \%)$ respondents whose location of practice was in a Tier 1 city, $86(22.3 \%)$ respondents in a Tier 2 city, $44(11.4 \%)$ respondents in a Tier 3 city, $38(9.8 \%)$ in a Tier 4 city, and $9(2.3 \%)$ in a Tier 5 city. The location of practice of $35(9.1 \%)$ respondents was unable to be classified (unclear or undetermined classification) according to the tier system.

\section{Years of clinical experience}

The mean number of years of clinical experience \pm SD was $16.48 \pm 9.42$ years (range: $1-45$ years), and the highest proportion of respondents was from those with between 10 and 19 years of clinical experience (144 respondents [39.0\%]). There were $86(23.3 \%)$ respondents with less than 10 years of clinical experience; 95 (25.7\%) respondents with between 20 and 29 years of clinical experience; and 44 (11.9\%) of respondents with 30 or more years of clinical experience. There were 17 (4.4\%) invalid inputs that were excluded.

Survey response analysis

The 5-point Likert scale responses to the questionnaire

Table 1. The mean \pm SD of the 5-Point Likert Scale Responses to the Questionnaire Items (Ranging from 1 being "Least Important" to 5 being "Most Important")

\begin{tabular}{|c|c|c|}
\hline & Mean & SD \\
\hline \multicolumn{3}{|l|}{ Modifiable risk factors } \\
\hline Use of hormone replacement therapy & 3.76 & 1.02 \\
\hline High level of stress ( $>50 \%$ of time) & 3.76 & 0.92 \\
\hline $\begin{array}{l}\text { Mental depression (at least of moderate } \\
\text { severity) }\end{array}$ & 3.69 & 0.98 \\
\hline Being overweight / obese & 3.58 & 1.02 \\
\hline No childbirth / First live birth after age 35 & 3.44 & 0.99 \\
\hline No breastfeeding & 3.24 & 1.18 \\
\hline Diet rich in meat / dairy products & 2.69 & 1.14 \\
\hline Lack of exercise ( $<3$ hours per week) & 2.67 & 1.07 \\
\hline Habit of drinking alcohol & 2.63 & 1.09 \\
\hline Diet rich in soy & 2.08 & 1.07 \\
\hline \multicolumn{3}{|l|}{ Non-modifiable risk factors } \\
\hline First-degree relative with breast cancer & 4.31 & 0.92 \\
\hline First-degree relative with ovarian cancer & 3.73 & 1.05 \\
\hline $\begin{array}{l}\text { Previous history of proliferative breast } \\
\text { disease with atypia }\end{array}$ & 3.72 & 1.04 \\
\hline $\begin{array}{l}\text { Beyond first-degree relative with breast } \\
\text { cancer }\end{array}$ & 3.46 & 0.94 \\
\hline High breast density & 3.31 & 0.97 \\
\hline Early menarche ( $<12$ years old $)$ & 3.26 & 1.09 \\
\hline $\begin{array}{l}\text { Beyond first-degree relative with ovarian } \\
\text { cancer }\end{array}$ & 3.07 & 1.02 \\
\hline Age $>65$ years & 2.88 & 1.08 \\
\hline $\begin{array}{l}\text { Previous history of proliferative breast } \\
\text { disease without atypia }\end{array}$ & 2.25 & 1.01 \\
\hline Being taller than average & 1.64 & 0.85 \\
\hline
\end{tabular}


items can be seen in Table 1. The five risk factors with the highest mean value (in descending order) were first-degree relative with breast cancer, use of hormone replacement therapy (HRT), high level of stress ( $>50 \%$ of time), firstdegree relative with ovarian cancer, and previous history of proliferative breast disease (PBD) with atypia. The five risk factors with the lowest mean value (in ascending order) were being taller than average, diet rich in soy, previous history of PBD without atypia, habit of drinking alcohol, and lack of exercise ( $<3$ hours per week).

The five risk factors with the highest proportion of responses being "Most Important" (in descending order) were first-degree relative with breast cancer, first-degree relative with ovarian cancer, use of HRT, previous history of PBD with atypia, and high level of stress $(>50 \%$ of time). The five risk factors with the lowest proportion of responses being "Most Important" (in ascending order) were being taller than average, previous history of PBD without atypia, diet rich in soy, habit of drinking alcohol, and lack of exercise ( $<3$ hours per week).

In addition, when asked if there is any other risk factor to include, the most common answers from the respondents were irregular sleep schedule $(n=17)$, mental health issues $(n=14)$, and poor sleep quality $(n=12)$.

\section{Subgroup analysis based on the medical specialty}

A statistically significant difference in responses for diet rich in soy was found $(\mathrm{p}=0.001)$, with a post-hoc analysis revealing that this factor was of a higher importance to medical oncologists than breast surgeons/ specialists (mean rank 210.31 vs. 165.34; $\mathrm{p}=0.004$ ). Likewise, significant differences in responses were also found for the use of HRT $(\mathrm{p}=0.022)$ and previous history of PBD without atypia $(p<0.001)$. Breast surgeons/specialists perceived a higher importance in the use of HRT as a risk factor than medical oncologists (mean rank 210.84 vs. 172.8; $\mathrm{p}=0.033$ ), and medical oncologists perceived a higher importance in the previous history of PBD without atypia as a risk factor than breast surgeons/specialists (mean rank 218.21 vs. $164.32 ; \mathrm{p}<0.001$ ).

Subgroup analysis based on the Chinese city tier system

Statistically significant differences in responses for lack of exercise $(p=0.042)$ and age $>65$ years $(p=0.031)$ were found. Physicians whose location of practice was in Tier 3 cities (mean rank 134.59) perceived lack of exercise as less important than physicians whose location of practice was in Tier 1 (mean rank 182.95; $\mathrm{p}=0.033$ ) or Tier 2 cities (mean rank 186.19; $\mathrm{p}=0.043$ ). Physicians whose location of practice was in Tier 2 cities perceived age $>65$ years as less important than physicians whose location of practice was in Tier 1 cities (mean rank 156.45 vs. $192.73 ; \mathrm{p}=0.048)$.

\section{Subgroup analysis based on the years of clinical experience}

Statistically significant differences in responses for diet rich in meat/dairy products $(p=0.003)$ and beyond first-degree relative with breast cancer $(p=0.006)$ were found. Physicians with $<10$ years of clinical experience (mean rank 155.29) perceived diet rich in meat/dairy products as less important than physicians with 20-29 years of clinical experience (mean rank 198.67; $p=0.029$ ) and physicians with $\geq 30$ years of clinical experience (mean rank 164.66; $\mathrm{p}=0.003$ ). Physicians with 20-29 years of clinical experience perceived beyond first-degree relative with breast cancer as less important than physicians with $<10$ years of clinical experience (mean rank 164.66 vs. 216.12; $\mathrm{p}=0.003$ ).

\section{Discussion}

Despite the improved understanding of known risk factors on breast cancer and the development of breast cancer risk assessment tools over the years, little is known about the physicians' perception towards these risk factors. As shown in this study, the breast cancer risk factors were not perceived as equally important, and the weighting of each risk factor also varied depending on the physician's medical specialty, location of practice, and the number of years of clinical experience.

There are several findings in this study that may have implications for improving breast cancer risk assessment strategies in China. The habit of drinking alcohol, which is known to increase risks of breast cancer and proliferative benign breast disease (Liu et al., 2015), was perceived to have a low-medium importance. This is especially concerning since the proportions of excessive drinking, frequent drinking, and binge drinking were reported to reach $51 \%, 7.8 \%$, and $26.6 \%$ among Chinese women, respectively ( $\mathrm{Li}$ et al., 2011).

Being overweight or obese was perceived to have a medium-high importance by the respondents, but the lack of exercise was perceived to be of low-medium importance. We suspect that the rapid development of China's economy and public transport infrastructure has given rise to more walkable cities, and thus its citizens became more physically active than before (Sun et al., 2021). Nonetheless, as the dietary pattern has become more westernized and obesity is increasingly becoming more prevalent in the Chinese population (Zhai et al., 2014), breast cancer risk prevention strategies should perhaps involve more interventions in this aspect.

High levels of stress and mental depression have become increasingly important and relevant as a breast cancer risk factor, with irregular sleep schedule, poor sleep quality, and mental health issues being the most commonly suggested non-traditional risk factors for breast cancer by respondents of this study. Since emerging evidence has suggested that there is a link between sleep difficulties and carcinogenesis (Owens et al., 2016; Trudel Fitzgerald et al., 2017), the inclusion of sleep habits in breast cancer risk assessment models may be warranted.

Lastly, even after considering that most of the respondents in this study were either breast surgeons/specialists or medical oncologists, the differences found in the weighting of the risk factors between different medical specialties, locations of practice (Chinese city tier system), and the number of years of clinical experience were particularly noteworthy. A possible reason for such differences could be due to the nature of their medical practice and the patient profiles that they see more often 
in their clinical practice. For example, surgeons tend to see more patients with early-stage breast cancer and oncologists tend to see more patients with late-stage breast cancer. Such differences suggest that the adoption of breast cancer risk assessment strategies would have to take into account the differences in physicians' perceptions toward individual risk factors.

Although the interpretation of this survey's findings was limited by the relatively low responses from physicians whose location of practice was in lower-tier cities, we believe that this study provides a more updated insight into the perceptions of physicians in China toward the breast cancer risk factors. These findings underline the potential improvements in breast cancer risk assessment strategies that can be done, particularly with the modifiable risk factors.

\section{Author Contribution Statement}

The authors confirm contribution to the paper as follows: study conception and design: LWCC; data collection: all authors; analysis and interpretation of results: all authors; draft manuscript preparation: LWCC, EFL. All authors reviewed the results and approved the final version of the manuscript.

\section{Acknowledgments}

We would like to thank the 386 anonymous physicians who contributed to the survey.

\section{References}

Feng RM, Zong YN, Cao SM, Xu RH (2019). Current cancer situation in China: good or bad news from the 2018 Global Cancer Statistics?. Cancer Commun (Lond), 39, 22.

Huang Y, Tong Z, Chen K, et al (2019). Interpretation of breast cancer screening guideline for Chinese women. Cancer Biol Med, 16, 825-35.

Li Y, Jiang Y, Zhang M, et al (2011). Drinking behaviour among men and women in China: the 2007 China Chronic Disease and Risk Factor Surveillance. Addiction, 106, 1946-56.

Liu Y, Nguyen N, Colditz GA (2015). Links between alcohol consumption and breast cancer: a look at the evidence. Womens Health (Lond), 11, 65-77.

Livaudais-Toman J, Karliner LS, Tice JA, et al (2015). Impact of a primary care based intervention on breast cancer knowledge, risk perception and concern: A randomized, controlled trial. Breast $J, 24,758-66$.

Owens RL, Gold KA, Gozal D, et al (2016). Sleep and breathing ... and cancer?. Cancer Prev Res (Phila), 9, 821-27.

Sun Z, Scott I, Bell S, Zhang X, Wang L (2021). Time distances to residential food amenities and daily walking duration: A cross-sectional study in two low Tier Chinese cities. Int $J$ Environ Res Public Health, 18, 839.

Trudel-Fitzgerald C, Zhou ES, Poole EM, et al (2017). Sleep and survival among women with breast cancer: 30 years of follow-up within the Nurses' Health Study. Br J Cancer, 116, 1239-46.

Zhai FY, Du SF, Wang ZH, et al (2014). Dynamics of the Chinese diet and the role of urbanicity, 1991-2011. Obes Rev, 15, 16-26.

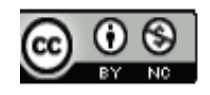

This work is licensed under a Creative Commons AttributionNon Commercial 4.0 International License. 